\title{
Factores asociados a la hipertensión arterial en el adulto mayor según la subregión
}

\author{
Nini J. García-Castañeda*, Doris Cardona-Arango, \\ Ángela M. Segura-Cardona y María O. Garzón-Duque
}

\author{
Facultad de Medicina. Universidad CES, Medellín, Colombia
}

Recibido el 20 de noviembre de 2015; aceptado el 5 de febrero de 2016

Disponible en Internet el 20 de marzo de 2016

\author{
PALABRAS CLAVE \\ Hipertensión; \\ Adultos; \\ Prevalencia; \\ Calidad de vida; \\ Atención primaria
}

\begin{abstract}
Resumen
Introducción: El envejecimiento de la población y la hipertensión arterial presentaron una tendencia al aumento en las subregiones del Departamento de Antioquia.

Objetivo: Determinar los factores asociados a la hipertensión arterial en el adulto mayor, según las subregiones del Departamento de Antioquia en el segundo semestre del año 2012.

Método: Estudio observacional, descriptivo, transversal y retrospectivo donde se caracterizaron los factores asociados a la hipertensión arterial. Se usó una fuente secundaria de información utilizando la base de datos del estudio "Situación de salud y condiciones de vida del adulto mayor, Departamento de Antioquia, 2012".

Resultado: Se analizaron 4.248 encuestas diligenciadas por los adultos mayores, el 65,5\% fueron mujeres hipertensas, el 64,1\% adultos mayor joven (60 a 74 años de edad), la subregión de Oriente ( $R P=1,67$, IC al 95\%: 1,27-2,19), la pobreza se asocia con la enfermedad, el estrato socioeconómico cero ( $R P=2,54$, IC al 95\%: $0,85-7,57)$, se encontró asociación con los hábitos como: el consumo de alcohol, el tabaco y el estado nutricional. El 21,8\% presentó prevalencia de la diabetes y el $28,3 \%$ de los adultos hipertensos manifestó que empeorará mucho la calidad de vida.

Conclusiones: La condición de ser: adulto mayor viejo, sexo femenino, afrocolombiano, residir en estrato cero y presentar un estado nutricional moderado, diabetes, problemas del corazón y cerebro, se asocia con la hipertensión arterial. Se requiere la implementación de las siete $P$ (7P) de la salud pública: las políticas públicas, la planeación en salud, la población vulnerable, la promoción de la salud, la prevención del riesgo, la participación comunitaria y los principios éticos.

(c) 2016 Sociedad Colombiana de Cardiología y Cirugía Cardiovascular. Publicado por Elsevier España, S.L.U. Este es un artículo Open Access bajo la licencia CC BY-NC-ND (http:// creativecommons.org/licenses/by-nc-nd/4.0/).
\end{abstract}

\footnotetext{
* Autor para correspondencia.

Correos electrónicos: ninijohannagarcia@gmail.com, ninijohanna.garcia@antioquia.gov.co (N.J. García-Castañeda).
} 


\section{KEYWORDS}

Hypertension;

Adults;

Prevalence;

Quality of life;

Primary care

\section{Factors associated to arterial hypertension in older adults according to subregion}

\begin{abstract}
Introduction: Ageing population and arterial hypertension showed a rising trend in the subregions of the Antioquia department.

Objective: To determine factors associated to arterial hypertension in old adults according to the subregion of the Antioquia department during the second semester of the year 2012 .

Method: Cross-sectional, descriptive, observational, retrospective study where factors associated to arterial hypertension were identified. A secondary source of information was used, with the database of the study Health situation and living conditions of old adults, Antioquia department, 2012 ("Situación de salud y condiciones de vida del adulto mayor, Departamento de Antioquia, 2012"').

Results: 4,248 surveys completed by old adults were analysed, $65.5 \%$ were hypertensive women, $64.1 \%$ were young elderly (between 60 and 74 years old), Oriente subregion $(\mathrm{OR}=1.67, \mathrm{Cl} 95 \%)$ : 1.27-2.19), poverty is associated to illness, zero socioeconomic status (OR $=2.54, \mathrm{Cl} 95 \%$ : 0.85 7.57), an association was found with alcohol and tobacco consumption and nutritional status. $21.8 \%$ showed diabetes prevalence and $28.3 \%$ of hypertensive adults expressed that it worsened their quality of life.

Conclusions: Being an older adult, female, of Afro-Colombian origin, living in status zero and showing a moderate nutritional status, diabetes, cardiac or brain problems are associated with arterial hypertension. Implementing the 7 public health indications is required: public policies, health planning, vulnerable population, health promotion, risk prevention, community participation and ethical principles.

(c) 2016 Sociedad Colombiana de Cardiología y Cirugía Cardiovascular. Published by Elsevier España, S.L.U. This is an open access article under the CC BY-NC-ND license (http:// creativecommons.org/licenses/by-nc-nd/4.0/).
\end{abstract}

\section{Introducción}

La hipertensión arterial es un síndrome que incluye no solo: la elevación de las cifras de la presión arterial, tomada con una técnica adecuada, sino los factores de riesgo cardiovascular modificables o prevenibles y no modificables; los primeros abarcan los metabólicos (la dislipidemia, la diabetes y la obesidad), los hábitos como el consumo de cigarrillo, el sedentarismo, la dieta y el estrés, y actualmente, se considera la hipertrofia ventricular izquierda; los segundos (la edad, el género, el grupo étnico, la herencia). La hipertensión es una enfermedad silenciosa, lentamente progresiva, que se presenta en todas las edades con preferencia en las personas entre los 30 y 50 años de edad, generalmente, asintomática que después de 10 a 20 años ocasiona daños significativos en el órgano blanco ${ }^{1}$.

La prevalencia creciente de la hipertensión arterial se atribuye: al aumento de la población, a su envejecimiento y a factores de riesgo relacionados con el comportamiento, como: la dieta malsana, el uso nocivo del alcohol, la inactividad física, el sobrepeso o la exposición prolongada al estrés $^{2}$. En Colombia, el Estudio Nacional de Factores de Riesgo de Enfermedades Crónicas - ENFREC ॥, realizado en el año 1998, arrojó una prevalencia de la hipertensión arterial de $12,3 \%$. En cuanto a la prevalencia de la población adulta con hipertensión arterial, según el grupo de edad de 60 a 64 años el $19,6 \%$ y de 65 a 69 años el $20,6 \%{ }^{3}$.

Según se prevé, el porcentaje de las personas de 60 y más años de edad en todo el mundo se duplicará entre el año 2000 y el 2050 y pasará del 10 al 21\%. El envejecimiento de la población pasará a ser una cuestión de primordial importancia en los países en desarrollo que, según se proyecta, envejecerán rápidamente en la primera mitad del siglo xxI. Se espera que para el año 2050 el porcentaje de personas de edad aumentará del 8 al 19\%, mientras que el de niños descenderá del 33 al $22 \%$. Este cambio demográfico plantea un problema importante en materia de recursos ${ }^{4}$.

En la investigación de "Situación de salud y condiciones de vida del adulto mayor, departamento de Antioquia, 2012"', se encontró que la enfermedades crónicas siguen siendo de las patologías que más aquejan los adultos mayores, estando en primer lugar la hipertensión arterial, seguida de la diabetes, los problemas articulares, los pulmonares y otros. En Antioquia, dos de cada tres adultos mayores, el $59,7 \%$, han sido diagnosticados con hipertensión arterial ${ }^{5}$.

Si no se aborda a tiempo, el problema de la hipertensión esta tendrá importantes repercusiones económicas y sociales: la muerte prematura, la discapacidad, las dificultades personales y familiares, la pérdida de ingresos y los gastos médicos ocasionados por la hipertensión arterial repercuten en las familias, las comunidades y las finanzas nacionales ${ }^{2}$.

Es importante identificar las variables asociadas a la hipertensión arterial en el adulto mayor en el Departamento de Antioquia por ser una de las primeras causas de la morbilidad y la mortalidad y que permita un abordaje desde la atención primaria en salud al adulto mayor y articular las acciones con la política pública del adulto mayor. Por lo anterior este estudio determinó los factores: demográficos, económicos, hábitos y calidad de vida asociados con la hipertensión arterial en el adulto mayor, según la subregión 
del departamento de Antioquia en el segundo semestre del año 2012.

\section{Método}

Se diseñó un estudio analítico observacional de tipo descriptivo transversal, donde se observaron: los factores demográficos, los económicos, los hábitos y la calidad de vida que pudieron estar asociados a la hipertensión arterial en la población adulta mayor residente en las nueve subregiones del departamento de Antioquia y la ciudad de Medellín en el año 2012. El estudio se revisó y se aprobó por parte del Comité de Ética de la Universidad del CES, dado que el análisis de la base de datos no representaba riesgo para los participantes. Toda la información se manejó de forma confidencial según las recomendaciones de la Resolución 8430 de 1993 del Ministerio de Salud ${ }^{6}$ y la Declaración de Helsinki de la Asociación Médica Mundial ${ }^{7}$.

Este análisis se llevó a cabo con fuentes secundarias de información de la base de datos implementada por la Universidad CES para el proyecto "Situación de salud y condiciones de vida del adulto mayor en el departamento de Antioquia, 2012 " 5 . La población estuvo constituida por 4.248 registros de los adultos mayores que fueron seleccionados mediante muestreo probabilístico y polietápico y los resultados fueron expandidos a 654.474 adultos mayores del departamento de Antioquia.

El instrumento de recolección utilizado para el estudio marco fue diseñado por investigadores de los grupos de Epidemiologia y Bioestadística y Observatorio de la Salud Pública de la Universidad CES, responsables del mismo. Se construyó una base de datos para este estudio que incluyó: variables demográficas (la subregión, la edad, el sexo, el estado civil, la etnia, el área de la residencia), variables económicas (el nivel de escolaridad, el ingreso económico, la ocupación, el estrato socioeconómico, el tipo de vivienda, la percepción de la situación económica), hábitos (el tiempo libre, el consumo de cigarrillo, el consumo de alcohol, el estado nutricional y las comorbilidades como: la diabetes, los problemas del corazón y cerebrales, el tipo de tratamiento) y la calidad de vida.

El control de sesgos fue supeditado al control que se hizo en el estudio marco ${ }^{5}$ y se procedió nuevamente a revisar y verificar que los datos estuvieran completos para cada variable a utilizar en este estudio, teniendo en cuenta aquellas en la que pudiera presentarse alguna inconsistencia con el fin de reportar dicha información en los resultados y la discusión del estudio.

Se realizó el análisis univariado donde se describieron cada una de las variables objeto del estudio. Posteriormente, se efectuó el análisis bivariado donde se establecieron las asociaciones, donde se tomaron las variables que por su comportamiento estadístico $(p<0.25)$ y por la literatura podrían aportar a la explicación de la hipertensión arterial en el adulto mayor, se utilizó la prueba de asociación Chi2 para el cruce de la variable dependiente, la hipertensión arterial con las variables independientes y el análisis multivariado; se llevó a cabo un modelo de regresión logística explicativo con la construcción del método Enter, con el fin de determinar cuáles variables aportaron más de manera conjunta a la explicación de la hipertensión arterial en los adultos mayores del departamento de Antioquia para el año 2012. Todos los cálculos estadísticos se realizaron en el programa SPSS 20.0 Licencia de la Universidad CES.

\section{Resultados}

Se hizo un análisis multivariado mediante la regresión logística binaria donde se ingresaron todas las variables demográficas, económicas, hábitos y calidad de vida que en el análisis bivariado obtuvieron un valor de $\mathrm{p}<0,25$ según el criterio de Hosmer-Lemeshow.

En la variable subregión en la tabla 1, la subregión Oriente RP ajustado: 1,56, Norte RP ajustado: 1,26 y el Nordeste RP ajustado: 1,02 como las tres regiones que más reunieron condiciones para que los adultos mayores se enfermaran de la hipertensión arterial, comparadas con la ciudad de Medellín.

En cuanto a la edad, se observaron los adultos mayores viejos (75 a 89 años), fueron los que más desarrollaron la hipertensión arterial comparados con los jóvenes (60 a 74 años), cuando se ajustó por las demás variables. (RP crudo $=1,73$ y RP ajustado =1,59 IC: 1,201 - 2,105).

Las mujeres mayores de 60 años de edad, del departamento de Antioquia (RP ajustado=1,42 IC: 1,093 - 1,855), fueron 0,42 veces más hipertensas que los hombres cuando se ajustó por las variables: demográficas, económicas, hábitos y calidad de vida.

Se observaron cambios importantes en la variable estado civil, los adultos mayores en unión libre donde en el RP crudo: 2,24 sobrestimó la asociación RP ajustado: 3,54, dejándolo como el estado de mayor prevalencia de la hipertensión arterial 2,54 veces más en los adultos en unión libre que en aquellos divorciados cuando se ajustó por las demás variables.

En la variable etnia no se encontró una asociación estadísticamente significativa $(p=0,152)$, no obstante, se analizó en el modelo explicativo por cumplir con un valor de $p<0,25$ según el criterio de Hosmer-Lemeshow, se evidenció como los adultos mayores afrocolombianos tienden a tener 1,64 veces más prevalencia de hipertensión arterial con relación a los indígenas cuando se ajustó por las demás variables $(R P$ crudo $=2,16$ y RP ajustado =2,64 IC: 0,394 $-17,756)$.

En cuanto al nivel de escolaridad, los adultos que cursaron secundaria completa e incompleta y técnico o tecnólogo completo e incompleto RP crudo: 1,66 y 1,63 sobreestimó la asociación RP ajustados: 2,35 y 3,5, se observó cómo los adultos mayores con secundaria tuvieron 1,35 veces y los técnicos 2,5 veces más prevalencia de la hipertensión con relación a los universitarios cuando se ajustó por las demás variables.

Para el caso del estrato socioeconómico de la vivienda se encuentran grandes cambios, el estrato cero RP crudo: 2,54 sobreestimó la asociación RP ajustado: 20,62 , lo que evidenció el alto riesgo de los adultos mayores que presentaron un estrato económico cero tienden a presentar más la enfermedad hipertensiva en comparación a los que no tienen un estrato asignado ajustado por las demás variables.

Los hábitos que se exploraron fueron: el consumo de cigarrillo y las bebidas alcohólicas, el estado nutricional, el tratamiento farmacológico, la diabetes, los problemas del corazón y los problemas cerebrales. 
Tabla 1 Variables demográficas, económicas, hábitos y calidad de vida, que más aportan a la explicación de la hipertensión arterial en los adultos mayores del departamento de Antioquia para el año 2012

\begin{tabular}{|c|c|c|c|c|c|c|}
\hline & \multirow[t]{2}{*}{ RP crudo } & \multicolumn{2}{|c|}{ IC $95 \%$} & \multirow[t]{2}{*}{ RP ajustado } & \multicolumn{2}{|c|}{ IC 95\% } \\
\hline & & Inferior & Superior & & Inferior & Superior \\
\hline \multicolumn{7}{|l|}{ Variables Demográficas } \\
\hline \multicolumn{7}{|l|}{ Subregión } \\
\hline Medellín & 1 & - & - & 1 & - & - \\
\hline Bajo Cauca & 1,2 & 0,919 & 1,556 & 0,42 & 0,224 & 0,779 \\
\hline Magdalena Medio & 1,13 & 0,872 & 1,465 & 0,61 & 0,333 & 1,106 \\
\hline Nordeste & 1,38 & 1,063 & 1,8 & 1,02 & 0,552 & 1,881 \\
\hline Norte & 1,36 & 1,038 & 1,779 & 1,26 & 0,644 & 2,479 \\
\hline Occidente & 1,41 & 1,077 & 1,848 & 0,61 & 0,321 & 1,156 \\
\hline Oriente & 1,67 & 1,273 & 2,194 & 1,56 & 0,745 & 3,268 \\
\hline Suroeste & 1,37 & 1,051 & 1,786 & 0,72 & 0,39 & 1,319 \\
\hline Urabá & 1,13 & 0,864 & 1,465 & 0,36 & 0,18 & 0,71 \\
\hline Valle de Aburra & 1,35 & 1,034 & 1,768 & 0,84 & 0,457 & 1,536 \\
\hline \multicolumn{7}{|l|}{ Edad } \\
\hline Adulto Mayor Joven (60-74) & 1 & - & - & 1 & - & - \\
\hline Adulto Mayor Viejo (75-89) & 1,73 & 1,509 & 1,993 & 1,59 & 1,201 & 2,105 \\
\hline Adulto Mayor Longevo (90-99) & 1,3 & 0,816 & 2,073 & 0,73 & 0,297 & 1,773 \\
\hline Adulto Mayor Centenario $(>=100)$ & 0,45 & 0,112 & 1,8 & 0,61 & 0,017 & 21,905 \\
\hline \multicolumn{7}{|l|}{ Sexo } \\
\hline Hombre & 1 & - & - & 1 & - & - \\
\hline Mujer & 1,46 & 1,288 & 1,659 & 1,42 & 1,093 & 1,855 \\
\hline \multicolumn{7}{|l|}{ Estado civil } \\
\hline Divorciado & 1 & - & - & 1 & - & - \\
\hline Soltero & 2,67 & 1,013 & 7,022 & 2,53 & 0,312 & 20,626 \\
\hline Casado & 3,13 & 1,196 & 8,182 & 3 & 0,372 & 24,248 \\
\hline Viudo & 3,86 & 1,473 & 10,115 & 2,79 & 0,343 & 22,742 \\
\hline Separado & 2,13 & 0,798 & 5,676 & 1,7 & 0,205 & 14,163 \\
\hline Unión Libre & 2,24 & 0,836 & 5,985 & 3,54 & 0,427 & 29,397 \\
\hline \multicolumn{7}{|l|}{ Etnia } \\
\hline Indígena & 1 & - & - & 1 & - & - \\
\hline Afrocolombiano & 2,16 & 0,774 & 6,039 & 2,64 & 0,394 & 17,756 \\
\hline Raizal & 0,33 & 0,031 & 3,589 & 0 & 0 & . \\
\hline Mestizo & 2,21 & 0,799 & 6,099 & 2,21 & 0,337 & 14,502 \\
\hline Otro & 1,8 & 0,508 & 6,353 & 1,56 & 0,101 & 23,981 \\
\hline \multicolumn{7}{|l|}{ Área de Residencia } \\
\hline Rural & 1 & - & - & 1 & - & - \\
\hline Urbana & 1,14 & 0,91 & 1,426 & 0,8 & 0,494 & 1,287 \\
\hline \multicolumn{7}{|l|}{ Variables Económicas } \\
\hline \multicolumn{7}{|l|}{ Nivel de Escolaridad } \\
\hline Sin Educación & 1,97 & 1,132 & 3,427 & 2,09 & 0,506 & 8,672 \\
\hline Primaria completa e incompleta & 2,06 & 1,193 & 3,55 & 1,93 & 0,474 & 7,842 \\
\hline Secundaria completa e incompleta & 1,66 & 0,943 & 2,938 & 2,35 & 0,561 & 9,863 \\
\hline $\begin{array}{l}\text { Técnico o tecnólogo completo } \\
\text { e incompleto }\end{array}$ & 1,63 & 0,8 & 3,329 & 3,5 & 0,584 & 20,985 \\
\hline $\begin{array}{l}\text { Universitario Posgrado completo } \\
\text { e incompleto }\end{array}$ & 1 & - & - & 1 & - & - \\
\hline \multicolumn{7}{|l|}{ Ingreso económico en el mes anterior } \\
\hline Si tuvo ingresos & 1 & - & - & 1 & - & - \\
\hline No tuvo ingresos & 1,14 & 1,005 & 1,287 & 0,96 & 0,735 & 1,245 \\
\hline \multicolumn{7}{|l|}{ Estrato socioeconómico de la vivienda } \\
\hline Estrato 0 & 2,54 & 0,85 & 7,573 & 20,62 & 1,43 & 297,28 \\
\hline Estrato 1 & 1,15 & 0,763 & 1,726 & 0,64 & 0,325 & 1,28 \\
\hline Estrato 2 & 1,18 & 0,791 & 1,765 & 0,75 & 0,376 & 1,5 \\
\hline Estrato 3 & 1,42 & 0,933 & 2,155 & 0,89 & 0,404 & 1,962 \\
\hline Estrato 4 & 0,7 & 0,419 & 1,177 & 0,29 & 0,1 & 0,839 \\
\hline Estrato 5 & 0 & 0 & . & & & \\
\hline No asignado & 1 & - & - & 1 & - & - \\
\hline \multicolumn{7}{|l|}{ Situación económica actual } \\
\hline Mala & 1,48 & 0,821 & 2,655 & 1,56 & 0,369 & 6,603 \\
\hline Regular & 1,67 & 0,942 & 2,969 & 1,82 & 0,445 & 7,462 \\
\hline
\end{tabular}


Tabla 1 (Continuación)

\begin{tabular}{|c|c|c|c|c|c|c|}
\hline & \multirow[t]{2}{*}{ RP crudo } & \multicolumn{2}{|c|}{ IC 95\% } & \multirow[t]{2}{*}{ RP ajustado } & \multicolumn{2}{|c|}{ IC 95\% } \\
\hline & & Inferior & Superior & & Inferior & Superior \\
\hline Bueno & 1,43 & 0,801 & 2,561 & 1,52 & 0,375 & 6,175 \\
\hline Muy bueno & 1,11 & 0,574 & 2,153 & 1,87 & 0,412 & 8,512 \\
\hline Excelente & 1 & - & - & 1 & - & - \\
\hline \multicolumn{7}{|l|}{ Hábitos } \\
\hline \multicolumn{7}{|l|}{ Consumo actual de cigarrillo } \\
\hline $\mathrm{Si}$ & 0,5 & 0,419 & 0,6 & 0,67 & 0,503 & 0,904 \\
\hline No & 1 & - & - & 1 & - & \\
\hline \multicolumn{7}{|l|}{ Consumo actual de bebidas alcohólicas } \\
\hline $\mathrm{Si}$ & 0,61 & 0,505 & 0,748 & 0,81 & 0,584 & 1,118 \\
\hline No & 1 & - & - & 1 & - & - \\
\hline \multicolumn{7}{|l|}{ Estado Nutricional } \\
\hline Buen Estado Nutricional (0-2) & 1 & - & - & 1 & - & \\
\hline Riesgo Nutricional Moderado (3-5) & 2,16 & 1,712 & 2,736 & 2,99 & 1,796 & 4,982 \\
\hline Riesgo Nutricional Alto $(>=6)$ & 2,49 & 2,036 & 3,056 & 2,42 & 1,486 & 3,939 \\
\hline \multicolumn{7}{|l|}{ Tratamiento Farmacológico } \\
\hline $\mathrm{Si}$ & 19,1 & 14,701 & 24,826 & 24,23 & 14,611 & 40,189 \\
\hline No & 1 & - & - & 1 & - & - \\
\hline \multicolumn{7}{|l|}{ Diabetes } \\
\hline $\mathrm{Si}$ & 2,42 & 2,022 & 2,896 & 1,26 & 0,886 & 1,784 \\
\hline No & 1 & - & - & 1 & - & - \\
\hline \multicolumn{7}{|l|}{ Problemas del corazón } \\
\hline $\mathrm{Si}$ & 2,6 & 2,096 & 3,23 & 1,89 & 1,297 & 2,742 \\
\hline No & 1 & - & - & 1 & - & - \\
\hline \multicolumn{7}{|l|}{ Problemas cerebrales } \\
\hline $\mathrm{Si}$ & 1,27 & 0,944 & 1,701 & 2,27 & 1,227 & 4,189 \\
\hline No & 1 & - & - & 1 & - & - \\
\hline \multicolumn{7}{|l|}{ Calidad de Vida } \\
\hline \multicolumn{7}{|l|}{ Calidad de vida actual } \\
\hline Mala & 2,02 & 1,054 & 3,884 & 0,51 & 0,11 & 2,318 \\
\hline Regular & 2,32 & 1,3 & 4,131 & 0,56 & 0,137 & 2,264 \\
\hline Bueno & 2,29 & 1,289 & 4,058 & 0,71 & 0,178 & 2,816 \\
\hline Muy bueno & 1,52 & 0,827 & 2,795 & 0,99 & 0,243 & 4,006 \\
\hline Excelente & 1 & - & - & 1 & - & - \\
\hline \multicolumn{7}{|l|}{ Estado de salud } \\
\hline Mala & 3,97 & 2,323 & 6,79 & 1,02 & 0,302 & 3,428 \\
\hline Regular & 4,17 & 2,579 & 6,728 & 1,21 & 0,395 & 3,688 \\
\hline Bueno & 2,9 & 1,801 & 4,679 & 1,2 & 0,401 & 3,59 \\
\hline Muy bueno & 1,78 & 1,065 & 2,98 & 1,14 & 0,362 & 3,579 \\
\hline Excelente & 1 & - & - & 1 & - & \\
\hline \multicolumn{7}{|l|}{ Satisfacción con su salud } \\
\hline Altamente insatisfecho & 1,97 & 1,178 & 3,307 & 1,34 & 0,399 & 4,506 \\
\hline Insatisfecho & 2,43 & 1,596 & 3,695 & 1,19 & 0,396 & 3,545 \\
\hline Satisfecho & 1,82 & 1,206 & 2,741 & 1,01 & 0,349 & 2,895 \\
\hline Muy satisfecho & 0,97 & 0,616 & 1,542 & 0,95 & 0,309 & 2,945 \\
\hline Altamente satisfecho & 1 & - & - & 1 & - & - \\
\hline Calidad de vida actual respecto a la & ce 10 años & & & & & \\
\hline Ha empeorado mucho & 1,21 & 0,945 & 1,539 & 0,9 & 0,502 & 1,605 \\
\hline Ha empeorado ligeramente & 1,33 & 1,048 & 1,694 & 1,24 & 0,702 & 2,195 \\
\hline Está igual & 1,02 & 0,804 & 1,288 & 0,86 & 0,497 & 1,477 \\
\hline Ha mejorado ligeramente & 1,05 & 0,813 & 1,363 & 1,02 & 0,576 & 1,82 \\
\hline Ha mejorado mucho & 1 & - & - & 1 & - & - \\
\hline Calidad de vida dentro de 10 años & & & & & & \\
\hline Empeorará mucho & 1,47 & 1,172 & 1,833 & 1,41 & 0,859 & 2,302 \\
\hline Empeorará ligeramente & 1,05 & 0,823 & 1,33 & 1,23 & 0,747 & 2,013 \\
\hline Sera igual & 1,08 & 0,862 & 1,35 & 1,42 & 0,876 & 2,298 \\
\hline Mejorará ligeramente & 1,1 & 0,877 & 1,39 & 1,18 & 0,744 & 1,875 \\
\hline Mejorará mucho & 1 & - & - & 1 & - & - \\
\hline
\end{tabular}


El consumo de cigarrillo en los adultos mayores que fuman RP crudo: 0,5 y RP ajustado 0,67 , se comportó como si disminuyera la ocurrencia de la hipertensión al observar el análisis bivariado arrojó un $18 \%$ de la prevalencia para los adultos fumadores hipertensos igual es el comportamiento con el consumo de bebidas alcohólicas RP crudo: 0,61 y RP ajustado 0,81 , los resultados del bivariado evidenciaron el $16 \%$ de los adultos mayores con el hábito actual de alcohol y con la hipertensión arterial.

En cuanto al estado nutricional se observó un incremento de los RP, evidenciando que el riesgo nutricional moderado RP crudo: 2,16 y RP ajustado 2,99 implicó que los adultos mayores con un riesgo nutricional moderado presentaron una tendencia a enfermarse más de la hipertensión arterial en comparación a los que tienen un buen estado nutricional.

La hipertensión arterial es un trastorno multifactorial y se incrementó en los adultos mayores del departamento cuando se relacionó con otras patologías, como se evidenció con la diabetes RP ajustado: 1,26, los problemas del corazón RP ajustado 1,89 y los problemas cerebrales RP ajustado: 2,27 , estas patologías se comportaron como un factor de riesgo que favorecieron la aparición de la hipertensión arterial en los adultos en comparación con los que no sufren estos eventos ajustado por las demás variables.

Para el año 2021 los adultos mayores del departamento de Antioquia percibirán que su calidad de vida no ha cambiado respecto a la actual RP crudo: 1,08 y RP ajustado: 1,42 y empeorará ligeramente RP crudo:1,05 y RP ajustado: 1,23 a nivel de salud pública se puede decir que la tendencia muestra que los adultos mayores que perciben su calidad de vida será igual o que ligeramente empeorará tienen mayor riesgo de presentar hipertensión arterial en relación con los adultos que manifiestan que su estado de salud mejorará mucho, ajustada a las demás variables: las demográficas, las económicas, los hábitos y la calidad de vida.

\section{Discusión}

En Colombia, según el informe técnico del Observatorio Nacional de Salud (ONS), para el año 2008 la prevalencia de la hipertensión arterial en la población general era el $22 \%^{8}$, según las guías latinoamericanas de la hipertensión arterial, sitúan a Colombia con una prevalencia de hipertensión de $23 \%$, en este mismo informe se reporta los cinco departamentos con mayor frecuencia: Antioquia, Valle del Cauca, Bolívar, Risaralda y Caldas, según los resultados de "Situación de salud y condiciones de vida del adulto mayor, departamento de Antioquia, 2012"' se observó que cada 3 adultos mayores encuestados presentó la hipertensión arterial ${ }^{5}$, de allí que surja el interés de estudiar en los adultos de 60 años de edad en adelante, la presencia de la hipertensión arterial y sus factores ya que es un evento que genera mayor carga de la enfermedad en el mundo, ocupando el primer lugar en cuanto a la morbilidad y la mortalidad $^{9}$. Teniendo el conocimiento que es una enfermedad que cada día va en aumento y que la población adulta mayor se ve expuesta se exploraron los siguientes factores: demográficos, económicos, hábitos y percepción de la calidad de vida.
Con respecto al área de residencia en el estudio, se tomó la población adulta mayor que residen en la zona urbana y centros poblados, las regiones que se encontraron con mayor porcentaje de adultos fueron: el Suroeste, el Valle de Aburrá, Medellín y en la zona rural correspondió al Occidente y al Oriente del departamento. Según la subregión donde más se concentraron los adultos mayores fue en Medellín $(52,8 \%)$, y al explorar el comportamiento de la hipertensión arterial las regiones del Oriente $(R P$ ajustado $=1,56)$, Norte $($ RP ajustado $=1,26)$ y el Nordeste $($ RP ajustado $=1,02)$ fueron las que presentaron la mayor prevalencia de la hipertensión arterial en la población adulta mayor.

Según el séptimo informe del Joint National Committe para: la prevención, la detección, la evaluación y el tratamiento de la hipertensión arterial (JNC 7), más de dos tercios de la población mayor de 65 años de edad, padece de hipertensión ${ }^{10}$. La edad promedio de los participantes del estudio correspondió a 70,4 años con una desviación estándar de 8,2 años, el grupo de adultos mayores viejos (75 a 89 años), son los que más presentan riesgo de la hipertensión ( RP ajustado $=1,59)$, este dato es congruente con los datos de prevalencia en distintos países ya que hay un marcado aumento de la enfermedad a edades más avanzadas, además, la variable edad tiene un papel muy importante en los modelos de estimación del riesgo cardiovascular total ${ }^{11}$, la edad es un factor no modificable que incrementa el riesgo de la hipertensión arterial, el riesgo se incrementa a partir de los 35 años de edad ${ }^{12}$.

Al analizar el sexo en el ॥ Estudio Nacional de Factores de Riesgo de Enfermedades Crónicas (ENFREC-II) arrojó una información respecto a que la hipertensión arterial es más frecuente en las mujeres y en los grupos de mayor edad ${ }^{3}$. En este estudio se evidenció que la prevalencia de la hipertensión es de $32 \%$ mayor en las mujeres adultas que en los hombres, en las mujeres adultas mayores la presión arterial aumenta más que en los hombres y continúa aumentando hasta los 80 años $^{13}$. Las mujeres que se enfrentan a esta enfermedad puede ser que nos les cause limitaciones inicialmente, pero deteriora su capacidad de desempeño, la hipertensión es el "enemigo silencioso que come por dentro", 14

Diversos estudios han demostrado niveles más altos de la presión arterial en la etnia negra, en los Estados Unidos la enfermedad hipertensiva es más frecuente en los individuos afroamericanos ${ }^{15}$, se observa en el estudio de los factores: demográficos, económicos, hábitos y calidad de vida asociados a la hipertensión arterial en el adulto mayor según la subregión en el departamento de Antioquia, se encontró que los adultos mayores afrocolombianos presentan 1,64 veces más el riesgo de presentar la hipertensión arterial, las regiones donde más se concentraron los afrocolombianos es el Urabá y el Bajo Cauca.

La hipertensión arterial está presente en el 35\% de todos los eventos cardiovasculares ateroscleróticos y en el $49 \%$ de todos los casos de la falla cardiaca e incrementa el riesgo de los eventos cerebrovasculares ${ }^{13}$. Los problemas del corazón que reportaron los adultos mayores del departamento de Antioquia presenta un comportamiento como factor de riesgo (RP ajustado $=1,89$ ) al igual que los problemas cerebrales (RP ajustado $=2,27$ ), se puede decir, que los adultos mayores hipertensos presentan un alto riesgo de presentar eventos cardiocerebrovasculares. 


\section{Conclusiones}

Este trabajo investigativo permitió concluir que en la población adulta mayor del departamento de Antioquia que presentó la hipertensión arterial correspondió a las mujeres, se encontró relación con los afrocolombianos, es una enfermedad que se concentró en los adultos que no tienen ingresos económicos y que residen en el estrato socioeconómico cero. El estado nutricional en riesgo alto, además, de los problemas del corazón, los problemas cerebrales son causas de la hipertensión arterial. Finalmente, se recomienda a las autoridades sanitarias la implementación de un conjunto de políticas y estrategias que denominamos como "Las $7 \mathrm{P}$ de la salud pública"':

1. Políticas públicas: implementación en los municipios de la Política Nacional de Envejecimiento y Vejez 20072019.

2. Planeación integral en salud: formulación de los Planes Territoriales de Salud con acciones en la dimensión de vida saludable y condiciones no transmisibles de acuerdo al Plan Decenal de Salud Pública 2012-2021.

3. Población vulnerable: dirigida a todos los adultos mayores de 60 años de edad.

4. Promoción de la salud: información de las estrategias de actividad física, control del tabaco, reducir el uso nocivo del alcohol.

5. Prevención del riesgo: mediante tamizajes de riesgo cardiovascular a toda la población.

6. Participación comunitaria: construcción de la política pública de envejecimiento y vejez y en la red de cabildo de adultos mayores.

7. Principios éticos: respeto por la autonómica, no maleficencia, beneficencia y justicia.

\section{Conflicto de intereses}

Los autores declaramos no tener ningún conflicto de intereses.

\section{Agradecimientos}

A la población adulta mayor que permitió ser entrevistada y a la Universidad CES, a la Gobernación de Antioquia, a la Secretaría Seccional de Salud y Protección Social de Antioquia.

\section{Bibliografía}

1. Ministerio de Salud y Protección Social. Guía 13. Guía de atención de la hipertensión arterial.

2. Organización Mundial de la Salud. Información general sobre la hipertensión en el mundo [Internet]. 2013 [citado 19 de marzo de 2015]. Recuperado a partir de: http://apps.who.int/iris/ bitstream/10665/87679/1/WHO_DCO_WHD_2013.2_spa.pdf?ua=1.

3. Ministerio de Salud, Dirección General de Promoción y Prevención. II Estudio Nacional de Factores de Riesgo de Enfermedades
Crónicas - ENFREC II [Internet]. SIVIGILA No. 47; 1999. Recuperado a partir de: http://www.col.ops-oms.org/iah/ indiceboletines1999.htm.

4. Informe de la segunda Asamblea Mundial sobre el Envejecimiento. En Naciones Unidas; 2002 [citado 31 de marzo de 2015]. Recuperado a partir de: http://www.un.org/es/ events/pastevents/ageing_assembly2/.

5. Cardona Arango Doris, Segura Cardona Ángela María, Garzón Duque María Osley. Situación de Salud y condiciones de vida del adulto mayor. Departamento de Antioquia 2012. Primera. Medellín, Colombia: CES; 2013.

6. Colombia M de S. Resolución No. 008430 de 1993. Por la cual se establecen las normas académicas, técnicas y administrativas para la investigación en salud. 1993.

7. WMA Declaration of Helsinki - Ethical Principles for Medical Research Involving Human Subjects [Internet]. 2013 [citado 3 de abril de 2015]. Recuperado a partir de: http://www.wma. net/es/30publications/10policies/b3/.

8. Informe técnico Observatorio Nacional de Salud. Carga de enfermedades por Enfermedades Crónicas No Transmisibles y Discapacidad en Colombia. [Internet]. [citado 12 de octubre de 2015]. Recuperado a partir de: http://www.ins.gov.co/ lineas-de-accion/ons/SiteAssets/Paginas/publicaciones/5to \%20Informe $\% 200 N S \% 20 v$-f1.pdf.

9. OMS. | Enfermedades cardiovasculares [Internet]. WHO. [citado 12 de octubre de 2015]. Recuperado a partir de: http://www. who.int/mediacentre/factsheets/fs317/es/.

10. Chobanian AV, Bakris GL, Black HR, Cushman WC, Green LA, Izzo JL, et al. Seventh report of the Joint National Committee on Prevention, Detection, Evaluation, and Treatment of High Blood Pressure. Hypertension. Diciembre de. 2003;42(6): 1206-52.

11. Giuseppe Mancia (Coordinador) (Italia)*, Robert Fagard (Coordinador) (Bélgica)*, Krzysztof Narkiewicz (Coordinador de Sección), (Polonia), Josep Redon (Coordinador de Sección) (España), Alberto Zanchetti, (Coordinador de Sección) (Italia), Michael Böhm (Alemania), Thierry Christiaens, (Bélgica), Renata Cifkova (República Checa), Guy De Backer (Bélgica), Anna Dominiczak, (Reino Unido), Maurizio Galderisi (Italia), Diederick E. Grobbee (Países Bajos), et al. Guía de práctica clínica de la ESH/ESC para el manejo de la hipertensión arterial (2013). 2013; 30. Recuperado a partir de: http://apps.elsevier.es/watermark/ctl_servlet?_f=10\&pident_ articulo=90265503\&pident_usuario=0\&pcontactid=\&pident revista $=67 \&$ ty $=75 \&$ accion $=$ L\&origen=zonadelectura\& $w e b=w w w$. elsevier.es\&lan=es\&fichero=67v30nSupl.3a90265503pdf001.pdf.

12. Ministerio de Salud y Protección Social, Colciencias. Guía de práctica clínica. Hipertensión arterial (HTA). Guía para profesionales de la salud. Guía No. 18 [Internet]. 2013. Recuperado a partir de: http://gpc.minsalud.gov.co/Documents/GuiasPDF-Recursos/HTA/GPC_Completa_HTA.pdf.

13. Guías Colombianas para el diagnóstico y tratamiento de la hipertensión arterial. SCC. febrero de 2007 [citado 2 de septiembre de 2015];13. Recuperado a partir de: http://scc.org. co/blog/2012/08/guias-colombianas-para-el-diagnostico-ytratamiento-de-la-hipertension-arterial/.

14. C MMB, Ch MS, M NT. Vivencia de la hipertensión arterial en la mujer adulta mayor: una experiencia silenciosa y en soledad. Cult Cuid. 5 de julio de 2011; 8(2):16-27.

15. Nardo CJ, Chambless LE, Light KC, Rosamond WD, Sharrett AR, Tell GS, et al. Descriptive Epidemiology of Blood Pressure Response to Change in Body Position The ARIC Study. Hypertension. 5 de enero de. 1999;33(5):1123-9. 